\title{
ASOCIACIONES DE MIGRANTES AFRICANOS Educación y formación
}

\author{
AFRICAN MIGRANT ASSOCIATIONS \\ Education and training \\ Josep M. PaLAUdÀrIAS I MARTí josep.palaudarias@udg.edu \\ Universidad de Girona. España
}

Carles Serra I Salamé carles.serra@udg.edu

Universidad de Girona. España

\begin{abstract}
RESUMEN
A partir de los datos obtenidos a través de un cuestionario suministrado a 206 representantes de asociaciones de inmigrantes de origen africano y de 20 historias de vida y 66 entrevistas realizadas a representantes de estas asociaciones, este artículo analiza la realidad educativa de las asociaciones desde diferentes puntos de vista. Primero, estableciendo el valor que los dirigentes asociativos dan a la educación (papel que ha jugado en sus procesos migratorios y papel que juega en las asociaciones que dirigen). En segundo lugar, aproximándonos a su nivel de estudios e itinerario formativo. $Y$ finalmente, analizando las actividades formativas que estas asociaciones impulsan o en las que participan (colaborando con otras asociaciones, instituciones 0 servicios). De estas actividades, se han analizado tanto su tipología (temas que se abordan: lengua, religión, inserción laboral, entre otros), como su orientación (tendencia al multiculturalismo y colaboración o distanciamiento respecto al ámbito educativo formal).
\end{abstract}

\section{Palabras Clave}

Educación; Formación; Inmigración; Multiculturalismo; Nivel de estudios; Organización.

\begin{abstract}
Based on the data obtained in a survey of 206 representatives of African immigrant associations, nine life histories and 54 interviews carried out with representatives of these associations, this article analyzes their educational reality from different points of view. First, we establish the importance the association leaders give to education (the role that education played in their migration processes and in the associations they lead). Second, we take a close look at their educational level and training. And finally, we analyze the training activities promoted by these associations or the activities in which they are involved (collaborating with other associations, institutions or services). Concerning these activities, we analyze their types (topics covered: language, religion, labor market integration, etc.), and orientation (tendency to multiculturalism and collaboration or distance from formal education institutions).
\end{abstract}

\section{KEYWORDS}

Education; Educational Level; Immigration; Multiculturalism; Organization; Training. 


\section{INTRODUCCIÓN}

Si la dinámica de las asociaciones de inmigrantes es una cuestión que no hace muchos años hemos empezado a analizar en el Estado español, la actividad formativa de estas asociaciones es un tema aún más novedoso, sobre todo si nos centramos en las asociaciones africanas, ya que hasta el momento esta cuestión no ha llamado la atención de gran parte de los investigadores que trabajan en el ámbito de las migraciones ${ }^{1}$. Nosotros con este artículo pretendemos realizar una primera aproximación a la realidad formativa de las asociaciones abordándola desde diferentes perspectivas.

La investigación marco en la que se ubica nuestro estudio se fijó cómo definir la estructura, las funciones y las necesidades de las asociaciones. Desarrollando este objetivo general, nosotros hemos centrado nuestro trabajo en realizar un análisis de la realidad formativa de las asociaciones fijándonos tres objetivos específicos. Un primer objetivo es estudiar el perfil formativo de los dirigentes de las asociaciones a través de su itinerario socioformativo y de su nivel de estudios, comparándolo con el nivel de estudios de la población inmigrante en general, y en particular con la originaria de África. Un segundo objetivo se ha concretado en el análisis de las actividades formativas que las asociaciones impulsan. Así pues, a lo largo del artículo vamos a establecer cómo hemos entendido la educación en el ámbito asociativo, cual es el valor que dan a la educación los dirigentes de las asociaciones, cuáles son sus itinerarios formativos y por último cuáles son las actividades de carácter formativo que organizan (o en las que participan) las asociaciones.

Como en el resto de artículos de este monográfico vinculados al proyecto de investigación "Asociacionismo e inmigración africana: funciones latentes y manifiestas", los datos cuantitativos proceden de 206 cuestionarios que han contestado asociaciones de inmigrantes africanos formadas por hombres y/o mujeres con origen en países africanos. De estos cuestionarios, 138 se han pasado en Cataluña, 54 en la Comunidad Valenciana y 14 en Navarra. Los datos cualitativos que aportamos son el fruto de analizar 66 entrevistas en profundidad que hemos realizado a hombres y mujeres representantes de asociaciones africanas y de 20 historias de vida realizadas también a representantes de asociaciones residentes en alguno de los tres territorios mencionados.

Este texto se inscribe dentro del proyecto de investigación "Asociacionismo e inmigración africana: funciones latentes y manifiestas", financiado como proyecto I+D del Ministerio de Ciencia y Tecnología en la convocatoria de 2008 (CSO2008-01122/SOCl).

${ }^{1}$ El interés por el asociacionismo inmigrante empezó a desarrollarse a finales de los años 90 [por ejemplo Crespo (1997) Garreta (1998)] y es sobre todo a partir del IV y V Congreso sobre la inmigración en España cuando aparecen más publicaciones sobre el tema. El asociacionismo abordado desde una perspectiva educativa aparece mucho más tarde [Soriano y González (2010) y Giró (2011)]. 
Para abordar el análisis de las actividades formativas en las asociaciones de inmigrantes debemos fijar los términos con los que vamos a trabajar. Entendemos por 'educación' cualquier práctica social humana a través de la cual adquirimos conocimientos y habilidades que nos permiten mantener, crear y recrear la cultura. Este concepto de educación nos lleva a diferenciar en la educación dos dimensiones: el sentido amplio y el sentido estricto.

La educación en el sentido amplio es aquella que implica un proceso global que promueve la interacción de la población implicada en el proceso educativo con la sociedad del entorno, es parte de la socialización. El sentido amplio de educación recoge las acciones y actividades educativas que se desarrollan independientemente de la existencia formal de instituciones educativas, poniendo de relieve que hay una amplia diversidad de agentes sociales que educan desde una perspectiva no formal o informal. Estos agentes tienen una gran incidencia en las dinámicas asociativas y en las posiciones que toman los asociados ante los retos que plantea la sociedad de acogida.

En este artículo, el sentido estricto de la educación lo entendemos como el proceso de enseñanza y aprendizaje que las asociaciones impulsan y que dirigen a los educandos a compartir y crear conocimiento y habilidades, así como a fomentar actitudes. Entendemos por 'formación' la transmisión y adquisición de habilidades y técnicas de actuación que unidas a los conocimientos aportados por la instrucción conducen a la educación. La educación a través de los procesos formativos transmite unas formas de pensar, de hacer y de comunicar que entendemos como un hecho político en cuanto la formación, según se oriente, realiza y transmite una determinada construcción de la historia y de la sociedad que oprime o empodera (Freire 1990; Giroux 2001).

Optar por este concepto de educación permite que nos aproximemos a la diversidad de actividades educativas que las asociaciones impulsan, todas ellas de carácter no formal y que tienen este doble sentido que antes apuntábamos, pues inciden en el proceso de desarrollo global de las asociaciones, de los asociados y de los beneficiarios de la formación por un lado y, por otro, recoge los aspectos más concretos de la experiencia de enseñanza y aprendizaje que los educandos experimentan.

Otra cuestión que queremos resaltar, y que es consecuencia de lo que hasta ahora hemos expuesto, es que, en el análisis de las actividades que llevan a cabo las asociaciones, hemos entendido como 'formación' una amplia serie de acciones, algunas de ellas con clara tradición didáctica, como por ejemplo la enseñanza de idiomas; pero también hemos entendido como acciones formativas otras actividades como las de sensibilización o las de promoción de proyectos de inserción laboral, ya que se implementan a través de procesos formativos y con técnicas de enseñanza y aprendizaje. 


\section{LA EDUCACIÓN EN EL PROYECTO MIGRATORIO DE LOS LÍDERES ASOCIATIVOS}

Para una parte de las personas entrevistadas (representantes o dirigentes de las asociaciones de inmigrantes de origen africano) la intención de completar su formación, el interés por cursar unos estudios que en sus países de origen no podían completar o el deseo de conseguir un trabajo acorde con la formación que hasta ese momento habían recibido, ha sido uno de los motivos que los ha impulsado a emigrar. Así lo manifiestan algunos de los dirigentes de asociaciones que hemos entrevistado: el representante de una asociación de inmigrantes guineanos explica que los déficits educativos de su país mueven a emigrar a todos aquellos que desean continuar con sus estudios ["La gente que está allí viene aquí a estudiar. Vamos, según sea, pero vienen a estudiar aquí" (AGUMV09)]. También el representante de una asociación de inmigrantes de origen nigeriano afirma²:

"Mi etnia siempre pone prioridad en educación. Yo estoy muy orgulloso de la zona de donde vengo de Nigeria, nadie, nadie de la zona de donde yo vengo que no tenga preparación, yo no soy el único. En Inglaterra, en Canadá, todos los profesiones de yoruba, de la etnia yoruba, ¿por qué? Porque queremos tener profesionales, queremos tener educación, y luego se transmite a los hijos, del abuelo al hijo, a Ios nietos y a aquella generación yoruba. Nosotros ponemos la parte de educación, quien tiene educación tiene cerebro, tiene conocimiento, por eso lo mejor es ir a la Universidad en Reino Unido, llegar a ir. Un yoruba llegar a ser doctor, y yo me voy a alegrar, puedo ser pobre pero el conocimiento nadie me lo van a quitar..." (ANIHV09).

Son muchos los dirigentes entrevistados que aluden a sus procesos formativos para explicar su proceso migratorio. No debemos extrapolar la importancia que la educación juega en estos procesos, pero sí debemos incorporarla como una variable que con frecuencia es desatendida y que el discurso social sobre "los inmigrantes" contribuye a ocultar. Sin pretender sobredimensionar tal variable, sí que pensamos que es necesario ponerla encima de la mesa: es una realidad que existe y que debemos analizar (como lo hacen aquellos autores que han reintroducido la vieja temática de la fuga de cerebros a la hora de analizar el impacto de los movimientos migratorios (Abella [2006], por ejemplo). ¿Cuáles son, pues, los perfiles formativos de los representantes de las asociaciones de inmigrantes de origen africano?

${ }^{2}$ Las grabaciones y transcripciones estarán a disposición de los investigadores que las soliciten. 


\section{Nivel DE ESTUdiOS DE LOS DIRIGENTES DE ASOCIACIONES DE MIGRANTES AFRICANOS}

"Yo hice económicas, pero al segundo año paré mis estudios, porque en Senegal tuvimos dos años blancos, porque quisimos mezclar la educación con lo político y eso estalló" (ASEHV10).

En las entrevistas encontramos muchos ejemplos de dirigentes o representantes de asociaciones de inmigrantes de origen africano que disponen de estudios universitarios. En la asociación de mujeres musulmanas An-Nur, por ejemplo, la mayor parte de las mujeres que componen la junta son tituladas, algunas con doctorado y solo unas pocas no poseen titulación. Pero son los datos obtenidos a través del cuestionario los que nos ofrecen una visión de conjunto de los perfiles formativos de los dirigentes asociativos. Sin embargo, antes de pasar al análisis de estos datos, consideramos pertinente situarlos en el contexto del conjunto de la población inmigrada y, más concretamente, de la de origen africano.

Para realizar esta contextualización hemos optado por consultar principalmente dos fuentes de información (Instituto Nacional de Estadística 2008; C. loé y Fernández 2010) que trabajan sobre los datos de la Encuesta Nacional de Inmigrantes del año 2007. Una lectura de los datos sobre el nivel de estudios de la población inmigrada, que exponemos en la Tabla 1, nos lleva a observar que el nivel formativo que mayoritariamente han alcanzado las personas de origen inmigrado es el de estudios secundarios. Si nos centramos en la población de 20 a 34 años los porcentajes de población que ha cursado educación secundaria y superior tienen un significativo aumento.

Tabla 1.

Nivel de estudios población inmigrada. Datos globales.

\begin{tabular}{ccc}
\hline Estudios Primarios & Estudios Secundarios & Estudios Terciarios \\
\hline $20 \%$ & $59 \%$ & $21 \%$ \\
\hline
\end{tabular}

Fuente: Colectivo loé y Fernández (2010).

Si nos centramos en la población originaria del continente africano, pues investigamos las asociaciones formadas por personas originarias de países africanos, observamos en la Tabla 2 que el perfil de estudios es inferior al antes mencionado para toda la población inmigrada, en particular para las mujeres. Los datos para la población que tiene entre los 20 y los 34 años no son mejores que los presentados en la mencionada tabla. A partir de los datos que hemos consultado podemos decir que "el colectivo según origen que presenta un menor nivel de estudios corresponde al continente africano, donde el grupo de 
Tabla 2.

Nivel de estudios de la población inmigrada de origen africano.

\begin{tabular}{cccc}
\hline Género & Estudios Primarios & Estudios Secundarios & Estudios Terciarios \\
\hline Hombres & $35 \%$ & $51 \%$ & $14 \%$ \\
Mujeres & $37 \%$ & $52 \%$ & $11 \%$ \\
\hline
\end{tabular}

Fuente: Colectivo loé y Fernández (2010).

inmigrantes sin estudios o con educación primaria es el más numeroso" —casi la mitad de los inmigrantes de este origen-(Instituto Nacional de Estadística 2008: 31).

Realizada la contextualización, presentamos a continuación los datos obtenidos a través del cuestionario sobre el nivel de estudios de los dirigentes de las asociaciones africanas de inmigrantes. Estos se presentan primero en su globalidad y posteriormente teniendo en cuenta diferentes variables: las zonas de origen, la composición por género de cada asociación y las tipologías de asociaciones que hemos construido para explicar las diferentes dinámicas de las asociaciones en cuanto a su organización.

\section{Nivel de formación de los dirigentes de asociaciones}

En los niveles inferiores de formación observamos que solo el 0,5\% no tiene estudios ni saben leer y escribir y que el 1,5\% dice que sabe leer y escribir. Los que han realizado estudios básicos pero no los han finalizado son el $0,5 \%$ de los encuestados y el $9,7 \%$ dice que ha finalizado los estudios básicos. En relación a los estudios secundarios observamos que el $32,5 \%$ expresa que ha alcanzado el nivel de bachillerato y el $9,2 \%$ ha estudiado formación profesional. Si nos centramos en los estudios universitarios los porcentajes también son altos: el 19,9\% dice tener un título de diplomatura y el $21,4 \%$ ha cursado una licenciatura. Si nos situamos en los estudios superiores de tercer ciclo, el 2,4\% expone que ha seguido estudios de doctorado y el 1,9\% ha cursado estudios de máster.

Estos datos globales nos indican que el nivel de formación de los representantes de las asociaciones africanas de inmigrados que han respondido la encuesta es superior al de toda la población africana y a toda la población inmigrada en relación a los estudios superiores. También debemos valorar que entre los dirigentes de asociaciones de inmigrados africanos están presentes niveles de formación muy escasos en un número muy reducido de dirigentes.

\section{Nivel educativo por zonas de origen}

Hemos diferenciado tres grupos de asociaciones según las zonas de origen: las formadas por personas norteafricanas, las formadas por personas de origen subsahariano y las mixtas (formadas por personas de ambos orígenes). 
Tabla 3.

Nivel de estudios de los responsables de asociaciones según la zona de origen de sus asociados.

\begin{tabular}{lccc}
\hline \multicolumn{1}{c}{ Nivel de estudios } & A. Norteafricanas & Mixtas & A. Subsaharianas \\
\hline Sin estudios y no sabe leer no escribir & - & - & 1,3 \\
Sabe leer y escribir & 1,6 & - & 2,6 \\
Básicos no finalizados & - & - & 1,3 \\
Básicos & 6,5 & 1,5 & 19,7 \\
Formación profesional & 4,8 & 7,4 & 14,5 \\
Bachillerato & 30,6 & 32,4 & 34,2 \\
Diplomatura Univ. & 27,4 & 25 & 9,2 \\
Licenciatura & 21 & 26,5 & 17,1 \\
Doctorado & 4,8 & 2,9 & - \\
Máster & 3,3 & 2,9 & - \\
Ns/Nc & - & 1,5 & - \\
\hline
\end{tabular}

Fuente: Elaboración propia, a partir de los datos obtenidos en el cuestionario.

Si comparamos los datos de los dirigentes de las asociaciones norteafricanas con los de los dirigentes de las asociaciones subsaharianas, observamos que en este último grupo los porcentajes son superiores en los niveles más bajos de formación y son claramente más bajos en los niveles universitarios. Pese a ello, más del $60 \%$ de los representantes encuestados tienen un nivel de formación igual o superior al de bachillerato (34'2\% nivel de bachillerato, $26^{\prime} 3 \%$ nivel universitario). Entre los representantes de las asociaciones norteafricanas el porcentaje de dirigentes con un nivel de bachillerato 0 superior es del $877^{\prime} 1 \%$ (bachillerato 30 ' $6 \%$, estudios universitarios 56 ' $5 \%$ ), cifras parecidas a las que se dan en las asociaciones mixtas (bachillerato $32 ' 4 \%$, estudios universitarios $57^{\prime} 3 \%$ ).

\section{Nivel educativo según la composición por género de las asociaciones}

Para analizar los datos en función del género hemos establecido tres categorías, las asociaciones formadas mayoritariamente por hombres, las asociaciones formadas mayoritariamente por mujeres y las mixtas, compuestas indistintamente por hombres y mujeres.

Como vemos, las representantes de las asociaciones de mujeres presentan unos datos que las alejan considerablemente del estereotipo de mujer africana inmigrada y sin estudios, ya que entre sus dirigentes el nivel inferior de formación que encontramos 
Tabla 4.

Nivel educativo según composición por género de las asociaciones.

\begin{tabular}{lccc}
\hline Nivel de estudios & Hombres & Mixtas & Mujeres \\
\hline Sin estudios y no sabe leer ni escribir & 0,6 & - & - \\
Sabe leer y escribir & 1,8 & - & - \\
Básicos no finalizados & 0,6 & - & - \\
Básicos & 11,1 & 0 & 9,5 \\
Formación Prof. & 9,9 & 5,5 & 4,8 \\
Bachillerato & 34,6 & 27,7 & 19 \\
Diplomatura & 20,4 & 11,4 & 23,8 \\
Licenciatura & 17,9 & 44,4 & 28,5 \\
Doctorado & 1,9 & 5,5 & 4,8 \\
Máster & 1,2 & 5,5 & 4,8 \\
Ns/Nc & - & - & 4,8 \\
\hline
\end{tabular}

Fuente: Elaboración propia, a partir de los datos obtenidos en el cuestionario.

es el de estudios básicos (el 9,5\%), en este mismo grupo los porcentajes de formación superior (diplomatura y licenciatura) son más altos que en las asociaciones de hombres. También situados en la formación superior, observamos que los niveles educativos más elevados están mucho más presentes entre las dirigentes que entre el conjunto de mujeres de origen africano. Por otro lado observamos que los niveles inferiores de formación que hemos detectado se concentran en las asociaciones de hombres, aunque también es en estas últimas asociaciones donde el porcentaje de dirigentes que ha cursado bachillerato es más alto. El porcentaje de los licenciados o licenciadas es más alto en las asociaciones mixtas.

\section{Nivel de estudios según la tipología de asociación}

Los datos obtenidos según las cuatro categorías de asociaciones que hemos establecido ${ }^{3}$ arrojan los siguientes porcentajes.

En las asociaciones que hemos clasificado como profesionalizadas, aquellas que presentan un nivel de organización elevado, tienen personal contratado y llevan más años funcionando, observamos que son mayoría los dirigentes que tienen estudios uni-

${ }^{3}$ El artículo de Garreta (2012) de este mismo número monográfico, expone con detalle las características de estas tipologías. 
Tabla 5.

Nivel de estudios según tipología de asociación.

\begin{tabular}{lcccc}
\hline Nivel de estudios & Profesionalizadas & $\begin{array}{c}\text { Voluntarista } \\
\text { de base }\end{array}$ & $\begin{array}{c}\text { Voluntarista } \\
\text { organizada }\end{array}$ & $\begin{array}{c}\text { Voluntarista } \\
\text { reticular }\end{array}$ \\
\hline Sin estudios y no sabe leer ni escribir & - & 1,1 & - & - \\
Sabe leer y escribir & - & 3,2 & - & - \\
Básicos no finalizados & - & 1,1 & - & - \\
Básicos & - & 16,8 & 7,0 & - \\
Formación Prof. & 9,1 & 8,4 & 7,0 & 14,0 \\
Bachillerato & 18,1 & 35,8 & 35,1 & 25,6 \\
Diplomatura & 36,4 & 14,6 & 14,0 & 34,9 \\
Licenciatura & 36,4 & 13,7 & 33,3 & 18,5 \\
Doctorado & - & 2,1 & 1,8 & 4,7 \\
Máster & - & 3,2 & 1,8 & - \\
Ns/Nc & - & - & - & 2,3 \\
\hline
\end{tabular}

Fuente: Elaboración propia, a partir de los datos obtenidos en el cuestionario.

versitarios (72,8\%). En este tipo de asociación es donde más alto es el porcentaje de dirigentes que han cursado estudios superiores.

Las asociaciones clasificadas como voluntaristas de base son las que en buena parte funcionan a través de voluntariado y en las que el nivel de organización es bajo. Esta tipología de asociación es la única que presenta niveles de formación bajos (el $4,3 \%$ no ha llegado a realizar estudios básicos). En esta tipología el nivel de estudios que presenta un porcentaje más alto es el de bachillerato $(35,8 \%)$. Los que han seguido estudios superiores en sus diferentes titulaciones son el 33,6\%.

Entre los dirigentes de las asociaciones que hemos categorizado de voluntarista organizada, que son aquellas que se organizan a través de comisiones y que muestran un nivel de consolidación importante, el nivel mínimo de formación es el de estudios básicos finalizados y los que han seguido el bachillerato también en esta tipología presentan el porcentaje más alto $(35,1 \%)$. Los que han cursado diferentes niveles de estudios superiores son el $50,9 \%$.

La cuarta tipología de asociación que hemos construido es la voluntarista reticular. Esta es una tipología de asociación que se caracteriza por un grado más importante de organización que las voluntaristas, aunque no destaca de forma clara por un mayor trabajo en comisiones y en asambleas. La característica más significativa de esta tipología es que su organización se dirige a la participación en redes y foros. En esta tipología, el nivel inferior de formación de sus dirigentes es de formación profesional (14\%) y los que 
han realizado estudios de bachillerato son el $25,6 \%$. Los que han alcanzado diferentes niveles de formación superior son el $58,1 \%$.

Una comparación entre las cuatro tipologías pone de relieve que si bien el nivel de estudios es superior en las asociaciones profesionalizadas, vemos que los niveles de formación superior son altos en las cuatro tipologías. En las asociaciones voluntaristas de base es donde aparecen niveles de estudios menos cualificados, situación que se refleja en una capacidad de organización más precaria según los datos que hemos recogido.

\section{TRAYECTORIAS EDUCATIVAS EN LOS PAÍSES DE DESTINO}

Hasta ahora hemos visto los niveles de estudios que se dan entre los dirigentes de las asociaciones de inmigrantes procedentes del continente africano y hemos visto que con relativa frecuencia se dan niveles de formación elevados. Los relatos de los líderes asociativos ponen encima de la mesa otra cuestión: su incorporación a nuestro país (y a nuestro mercado laboral) no siempre tiene en cuenta ese nivel formativo de partida. Como hemos visto, un $45^{\prime} 6 \%$ de las personas a las que se pasó el cuestionario, así como a muchas de las que posteriormente entrevistamos, tienen estudios universitarios. Pero si antes veíamos que la educación ocupaba un lugar importante en el relato migratorio de algunos de los representantes asociativos, podemos ver ahora como en muchos casos estos mismos representantes tienen serios problemas a la hora de finalizar sus propios estudios en el país de destino. Las causas esgrimidas por las personas entrevistadas que han vivido este tipo de trayectorias son diversas. Van desde la dificultad de compatibilizar los estudios con sus actividades laborales hasta los problemas que tienen cuando han de convalidar los títulos obtenidos en su país de origen ["No, imposible. Yo lo he intentado todo" afirma este representante de origen marroquí, "pero aquí he hecho unas formaciones, por ejemplo de agente de salud, un poquito de informática, un poquito de auxiliar de biblioteca" (AMAHN09)].

En otros casos (sobre todo entre las mujeres), se arguyen motivos familiares: la dificultad de mantener una casa, de ser madre y a la vez avanzar con los estudios. Uno de estos casos es el de la representante de una asociación senegalesa que estudió derecho en Francia y que tuvo que abandonar los estudios por motivos familiares, aunque posteriormente los retomó en España; esta vez como auxiliar de geriatría.

Un rasgo de las trayectorias académicas de aquellos que han conseguido reemprender sus estudios universitarios en España es el abandono de las carreras que habían iniciado en su país de origen, para pasarse a carreras propias del ámbito social. Es el caso de una mujer marroquí que abandonó los estudios de medicina que había empezado en su país por la diplomatura de trabajo social en España:

"Dejé medicina, o sea, ya no iba ni siquiera a presentarme, y empecé a hacer Trabajo Social. Empecé aquí, a hacer Trabajo Social. Era el segundo año, creo, que habían 
abierto. Hice Trabajo Social, tres años, tardé, trabajando. Acabé Trabajo Social y empecé a trabajar como trabajador social hasta la fecha" (AMAHV09I).

Podríamos citar también a todos aquellos que, una vez en nuestro país y metidos en "las cosas de la inmigración" han hecho cursos, postgrados y otros estudios en mediación, interculturalidad, etc. Un ejemplo claro de esta derivación hacia estudios del ámbito social relacionados con la inmigración lo encontramos en el relato de este hombre de origen marroquí:

"Mi formación es... Mi formación es... De primero tengo diplomatura en Ciencias Empresariales, luego un postgrado en la Gestión Global de la Inmigración de la Universidad de Rovira i Virgili, luego un máster en Valencia sobre la Cooperación y el Desarrollo, los movimientos Migratorios y Codesarrollo, y ahora estoy cursando otro máster y es los Estudios Culturales del Mediterráneo. Luego, en Francia, también estudié, pues, la Unión Europea, todo... Es como una formación amplia" (ASEHTA09).

Leyendo atentamente los relatos de los dirigentes y representantes asociativos, no podemos descartar que la orientación de algunas personas hacia la formación en titulaciones del campo social se vea favorecida por la existencia de un mercado de trabajo específico para inmigrantes (y preferiblemente para inmigrantes implicados en el funcionamiento de asociaciones, supuestamente predispuestos a influir en su comunidad o a trabajar para ella):

“- ¿Tú debes ser trabajador social de formación?

- Sí, bueno, de experiencia. Me han habilitado porque llevaba ocho años trabajando como educador social.

- ¿Pero no has estudiado?

- No, no.

- ¿No tienes estudios superiores?

- Claro, yo hice biología y acabé, cuando llegué aquí empecé farmacia, pero encontré que lo mío es lo social. Entonces, me he dedicado a la Coma, hice muchos proyectos ahí, el ayuntamiento [...] me ha contratado para coordinar un poco todo lo que es ocio y tiempo libre en la Coma" (AMAUHV10).

Los ejemplos aparecen con relativa frecuencia entre los representantes de asociaciones de las tres comunidades autónomas estudiadas: uno de los representantes de Senecat, una asociación senegalesa implantada en Cataluña, realizó estudios universitarios (sin finalizarlos) y en Cataluña ha dado continuidad a su formación participando en cursos sobre mediación; una mujer de Burkina Faso, representante de una asociación sociocultural de mujeres inmigradas (Endavant), en su país cursó bachillerato y dos cursos de formación superior como asistente de dirección, y en Cataluña ha cursado 
postgrados universitarios sobre mediación y sobre gestión de asociaciones y fundaciones; otro varón marroquí, que habló en nombre de la asociación multicultural Dar Salam cursó estudios de turismo y de empresariales en Marruecos, y en Cataluña ha hecho cursos de mediación; un dirigente de origen mauritano había hecho biología y en España inició farmacia, pero abandonó estos estudios por los de educador social; y el presidente de una asociación senegalesa de Navarra, habiendo cursado estudios en ciencias políticas y habiendo obtenido el doctorado, en la actualidad trabaja como mediador intercultural para la Cruz Roja.

\section{LA ORGANIZACIÓN DE ACTIVIDADES FORMATIVAS EN LAS ASOCIACIONES}

La mayoría de las asociaciones desarrollan actividades que, de un modo u otro, podemos considerar formativas. Estas actividades van desde la realización de talleres (de informática, de orientación laboral y para la búsqueda de empleo o de cuentos africanos), hasta la clase de idiomas (de origen 0 de destino $0^{4}$ ), y están dirigidas tanto a los mismos asociados (o a la "comunidad" a la cual representa la asociación), como al conjunto de la sociedad (se hacen actividades para las escuelas, en el municipio...) o incluso dirigidas a la población que continúa en el país de origen (en proyectos de codesarrollo). De hecho, a través de las entrevistas hemos podido constatar que la función educativa está entre los objetivos fundacionales de numerosas asociaciones: muchas nacen para dar respuesta a necesidades de aquellos que las fundan, y algunas de estas necesidades son educativas. Un ejemplo es Wafae, una asociación creada por estudiantes de origen marroquí y que desarrolla, ante todo, actividades de carácter educativo:

"Clases de castellano, la alfabetización para mujeres, de búsqueda de empleo, de apoyo y refuerzo escolar para los menores [...] Tenemos clases de castellano, alfabetización, apoyo y refuerzo escolar, alfabetización digital, orientación laboral, talleres de búsqueda de empleo, mediación intercultural" (AESMA10).

Junto a objetivos de carácter más político o social se expresa la voluntad de contribuir a la formación, en este caso de los jóvenes. Otro caso, es el de la Asociación Batama Kafo, que también aparece vinculado a un proyecto educativo, en este caso la construcción de una escuela en su país de origen:

“- ¿Entonces, cuál es la finalidad sobretodo de la asociación?

- Buscar algo para los niños del país porque puede salir un problema. Si tú no puedes trabajar allí no puedes hacer nada para emigrante. Tú tienes que estudiar allí, si no aprendes

\footnotetext{
${ }^{4}$ Entre los objetivos de este artículo no se encuentra el de analizar críticamente los discursos relativos a las "culturas de origen", ni el de cuestionar en qué medida lo que se lleva a cabo es un mantenimiento o bien una producción o recreación de aquello que los distintos agentes consideran que es "cultura" y es "de origen".
} 
algo no puedes hacer nada. Lo estamos haciendo por hacer una clase de escuela allí.

- Ah... vosotros ayudáis a los niños de allá.

- De allá. Ayudamos a los niños de allá. Porque mi mujer, los niños han nacido aquí, los niños tienen nacionalidad española. Todos son de escuela. Los que hay aquí no tienen ningún problema" (AMALHN10I).

La presencia de la educación entre los objetivos fundacionales de algunas asociaciones es algo común en las asociaciones islámicas que se crean en torno a mezquitas, y pretenden asegurar el conocimiento del árabe y de las prácticas y conocimientos islámicos. Esto es, por ejemplo, lo expresado por el representante de una asociación cultural islámica ("enseñar árabe para los niños, enseñar a escribir para los que no saben" (AMAHG10II). Es común que algunas asociaciones se creen para consolidar experiencias y actividades de carácter educativo que hasta ese momento se estaban desarrollando en mezquitas (Moreras 2009). En otros casos, la asociación nace con objetivos muy concretos, que no son propiamente de carácter educativo, pero rápidamente, a los objetivos fundacionales se incorporan otros objetivos que con frecuencia sí que son educativos. Un ejemplo es Aman, una asociación creada para luchar contra las mutilaciones genitales femeninas, que pronto acabó desarrollando otro tipo de actividades educativas. Otro caso es el de esta asociación de mujeres senegalesas:

"Mira, habíamos visto que había muchas mujeres que eran analfabetas, aunque estaban más tiempo aquí no manejaban bien el idioma. Como yo, no he ido nunca a una fiesta española, solamente hablar, el locutorio y ya está. No sabía sus culturas, no había interculturalidad. Además, las mujeres, por falta de manejar el idioma, no podían trabajar, tenían algunos maltratos" (ASEMV10).

Más allá de estos casos, lo más habitual es que los objetivos educativos aparezcan citados entre un repertorio de objetivos que puede ser bien diversos. En ocasiones, la diversidad de objetivos de estas asociaciones está en parte provocada por las demandas de la misma Administración, que pide a la asociación (o a los asociados) su colaboración en labores de mediación, apoyo escolar, codesarrollo, entre otros. Un caso, puede ser el de una asociación (AMAMT10I) que, no teniendo unas líneas de actuación bien definidas (más allá de trabajar para la promoción de la propia cultura), fácilmente se ve arrastrada por las demandas y las dinámicas propias de otras entidades o instituciones con las que colabora, hasta el punto de que su actividad acaba por abarcar desde las conferencias, el activismo social o las actividades de carácter identitario (orientadas al interior de la comunidad o hacia el exterior), hasta la colaboración con la universidad y la realización de investigaciones sobre las dificultades escolares de los menores miembros de su comunidad.

Evidentemente, "que se hagan muchas cosas" no está mal. Por otro lado es una dinámica que debemos comprender. Probablemente tiene sentido "hacer actividades" (expresado así, de forma genérica) dado que con frecuencia son estas actividades las que contribuyen a dinamizar la asociación, a potenciar la autoestima de los que se implican en ella, a potenciar su relación, incluso a empoderarlos (uno se siente más capa- 
citado si se le reconoce la capacidad de organizar actos). El despliegue de actividades tiene un sentido que va más allá de la "utilidad" explícita del acto que se organiza; hacer actividades (por diversas que estas sean) da vida a una asociación. Por otra parte, este despliegue contribuye al reconocimiento de la asociación por parte de las instituciones, las personas o las otras asociaciones del entorno. Uno no necesariamente es valorado por la coherencia y la eficacia de lo que hace, sino por su capacidad de hacerse presente en los más diversos contextos. Pensamos que esto es especialmente importante de destacar en relación a las asociaciones que se caracterizan por su carácter no profesional y voluntario. No deberíamos evaluar la actividad de tales asociaciones desde la perspectiva de la eficacia o la eficiencia en sus actuaciones, como si de organizaciones profesionales se tratara (pues la mayoría no lo son). Aunque sí que podemos constatar que el despliegue de actividades tan heterogéneas y la dispersión en iniciativas de tan diversa índole (incluso entre las asociaciones más pequeñas), puede tener sentido si entendemos la asociación simplemente como un grupo de personas dispuesto a trabajar, a cooperar, a hacer cosas en común (e incluso puede ser un modo eficaz para demostrar que se existe, que se está presente). Pero también debemos señalar que un despliegue tan disperso fácilmente puede restar eficacia al desarrollo de una línea de actuación concreta. En la medida, pues, que queramos considerar a las asociaciones como entidades o colectivos al servicio de un proyecto común, la acumulación de actividades, el sumarse a iniciativas de otros y el no trabajar persiguiendo objetivos bien definidos, resta capacidad a la acción asociativa. Y no solo capacidad: también coherencia y posibilidades de desarrollar una línea de trabajo reflexiva, con criterios bien definidos, y aumenta el riesgo de que la propia asociación pierda el rumbo y acabe siendo una pieza más al servicio de las instituciones. Es el riesgo que, de hecho, reconoce el representante de la asociación en la que se ha puesto en marcha un "aula de acogida" promovida por el gobierno valenciano y que facilita la concesión de los permisos de residencia (véase el apartado "actividades de acogida").

Es común entre las asociaciones más estructuradas que se creen áreas o comisiones de trabajo y que, entre estas, se cree la comisión de educación o comisiones que, de un modo u otro, tienen por objeto los temas eminentemente educativos que la asociación lleva a cabo. Este tipo de comisión está presente en el $35,6 \%$ de las asociaciones que se organizan a través de comisiones. Es en las asociaciones profesionalizadas donde tiene mayor presencia este tipo de comisión. El 50\% de estas asociaciones declara tener una comisión que se encarga de las cuestiones educativas. En las asociaciones de tipo voluntarista organizada este porcentaje disminuye, ya que el $36,8 \%$ dice tener una comisión de educación y en las de tipo voluntarista reticular el porcentaje baja al $25 \%$. Queremos incidir en que las asociaciones voluntaristas de base, que son las que no tienen ninguna comisión, son las que están dirigidas por personas que presentan los niveles de formación más precarios. También debemos tener en cuenta que en la creación de comisiones es muy importante el nivel de implicación de los asociados en las actividades que organizan las asociaciones. Un nivel de implicación que, como indican Aparicio y Tornos (2010: 73), es bajo, ya que "en un $53 \%$ de las asociaciones sus 
miembros no tienen ninguna o poca implicación. Solo en un $9 \%$ de ellas son mayoría los que tienen un nivel de implicación".

Aparicio y Tornos (op. cit.) en su estudio sobre las asociaciones de inmigrantes recogen las siete principales actividades que las asociaciones impulsan. Algunas de ellas tienen un claro carácter formativo, como el mantenimiento y difusión de la propia cultura y la promoción e integración del propio colectivo nacional. En nuestro estudio profundizamos sobre la diversidad de actividades formativas que las asociaciones dicen impulsar, ampliando de esta forma el estudio señalado anteriormente y poniendo de relieve que las asociaciones organizan una amplia gama de actividades formativas. Así, del análisis de los principales objetivos de las asociaciones se desprende que si, bien alguno de ellos parecen no tener una orientación educativa, en la práctica sí que muchos se implementan a través de actividades formativas. Este es el caso de apoyar a los inmigrados a nivel laboral, un objetivo que en la práctica se traduce en sesiones de formación que consisten, por ejemplo, en aprender a redactar un currículum (este objetivo es perseguido por el $40,3 \%$ de las asociaciones). Entre los objetivos con claro planteamiento educativo destacamos el mantenimiento de la cultura de origen, ya que para todos los tipos de asociaciones es el objetivo más perseguido (el $53,4 \%$ de los dirigentes de las asociaciones así lo manifiestan). Otro objetivo que nos parece interesante recoger, ya que sitúa la labor que realizan las asociaciones, es dar a conocer la lengua y la cultura del país de acogida a inmigrantes. Este es un objetivo importante para el $18,2 \%$ de las asociaciones profesionalizadas y para el $15,8 \%$ de las asociaciones voluntaristas estructuradas, mientras que para el resto de tipologías es un objetivo muy poco planteado.

\section{Principales actividades de carácter formativo}

Observamos que las asociaciones a través de sus actividades articulan tres niveles diferenciados de formación. Un primer nivel va dirigido a facilitar una mejor integración social y laboral de las personas de origen inmigrante en el país de acogida a través, por ejemplo, de la formación en las lenguas vehiculares del país o de la comunidad autónoma de acogida y de cursos de inserción laboral. Un segundo nivel va dirigido al mantenimiento de la cultura y la lengua de origen y también de las prácticas religiosas. En muchas ocasiones este nivel es el motivo que lleva a crear una asociación. Algunas asociaciones trabajan para que los nacionales, ya sea a través de actos festivos o de sesiones de formación, no pierdan sus raíces culturales y, sobre todo, que las llamadas segundas generaciones no se distancien de la cultura y prácticas religiosas que constituyen en buena parte el eje identitario de las primeras generaciones. De alguna manera este tipo de formación responde al objetivo de mantener la idea de comunidad en contraste con la comunidad de los otros. En el tercer nivel, las propias asociaciones se constituyen en actores formativos a través de las actividades de sensibilización externa que organizan, sea por encargo de la Administración local o por su participación en diversas campañas de sensibilización. 
La formación en las lenguas del país de acogida

Es una actividad de gran importancia para las asociaciones porque, para las personas inmigradas, el dominio de las lenguas habladas en el país de acogida es una cuestión de primera necesidad si quieren insertarse en el mundo laboral e integrarse en la sociedad en la que habitan ${ }^{5}$. Debemos tener en cuenta que un gran número de inmigrados tienen una lengua materna diferente del castellano y que por este motivo el aprendizaje de esta lengua forma parte de los primeros aprendizajes que inician los recién llegados. No obstante "unos 600.000 inmigrados no la leen y alrededor de 300.000 no la hablan" (Colectivo loé y Fernández 2010). Lo mismo sucede con las lenguas propias de las comunidades autónomas, aunque hay un número no desdeñable de inmigrados que las domina (el Colectivo loé y Fernández [op. cit] afirman que el catalán en alguna de sus variantes lo dominan 650.000 inmigrados y el euskera 16.000 inmigrados).

El aprendizaje de las lenguas propias de las comunidades de recepción tiene una significativa presencia en las actividades de las asociaciones. Se imparten clases para adultos y para menores, y en algunos casos solamente para hombres o bien solamente para mujeres.

Entre las asociaciones ubicadas en Cataluña, las clases de lengua catalana dirigidas solamente para adultos es la experiencia más común (14,5\%), seguidas de las clases que se imparten simultáneamente para adultos y menores $(7,2 \%)$. Pocas asociaciones imparten clases de catalán exclusivamente para menores. Podemos entender, entonces, que gran parte de las asociaciones considera suficiente el aprendizaje de esta lengua que los menores hacen en la escuela. Otras asociaciones organizan clases diferenciando si el alumnado es hombre $(0,7 \%)$ o mujer $(2,2 \%)$. En estos últimos casos las asociaciones organizadoras están formadas exclusivamente por hombres o por mujeres. Las asociaciones situadas en Cataluña también organizan clases de castellano para adultos y menores $(5,8 \%)$ o solamente para adultos $(8,7 \%)$.

En la Comunidad Valenciana las asociaciones imparten fundamentalmente clases en lengua castellana, ya sea para adultos o para menores $(20,4 \%)$ y exclusivamente para adultos en un 14,8\%. También se dan clases de catalán, valenciano, para adultos y menores, pero el porcentaje de asociaciones que organizan este tipo de actividad es inferior que el de las que organizan clases de castellano. Encontramos asociaciones que también imparten clases diferenciando si son hombres o mujeres. Las asociaciones que organizan clases solamente para mujeres son el $5,4 \%$ de las entrevistadas.

En Navarra el 28,6\% de las asociaciones imparten clases de castellano para adultos, no hay clases de castellano para menores. En la oferta no se diferencia entre hombres y mujeres. Ninguna asociación organiza clases en euskera.

\footnotetext{
${ }^{5}$ Sobre el papel que tiene el aprendizaje de las lenguas del país de recepción en los procesos de inserción, ver el artículo de Moncusí y Albert (2012) en este mismo número monográfico.
} 
Las asociaciones formadas principalmente por hombres son las que más actividades de formación en las lenguas de acogida realizan y, en relación con las zonas de origen de los asociados, las que menos actividades de este tipo realizan son las subsaharianas. Las diferentes tipologías de asociaciones que hemos construido en nuestra investigación generan formación en las lenguas del país de acogida, pero las profesionalizadas son las que más realizan este tipo de cursos, sobre todo en castellano. En relación con las lenguas de las comunidades autónomas las asociaciones voluntaristas organizadas son las que imparten más clases de catalán.

\section{Clases de religión}

Esta formación se realiza en asociaciones formadas por hombres o por mujeres, en las mixtas no se imparten. Por zonas de origen estas clases se dan en las asociaciones norteafricanas $(11,3 \%)$, mixtas $(8,8 \%)$ y subsaharianas $(5,3 \%)$. Las asociaciones voluntaristas organizadas son las que más imparten este tipo de formación. Las otras tipologías también la imparten aunque en menor porcentaje.

\section{Los proyectos de inserción laboral}

Usualmente estos proyectos implican sesiones de formación sobre cómo preparar un currículum y cómo orientar al alumnado para implementar estrategias con las que buscar un empleo. Los proyectos de inserción laboral están entre las cinco actividades que más llevan a cabo las asociaciones (sin duda por las necesidades laborales que en general presenta la población de origen inmigrado y por sus dificultades para conseguir un contrato de trabajo). Desde la perspectiva de género, las únicas asociaciones que no organizan este tipo de actividad son las formadas solo por mujeres y se organizan más en asociaciones formadas solamente por hombres que en el resto de asociaciones. Si dividimos a las asociaciones según las zonas de origen, observamos que se organizan en las tres tipologías de asociación, pero más en las norteafricanas (29\%).

Las asociaciones profesionalizadas concentran buena parte de sus esfuerzos en generar proyectos de inserción laboral (45,5\%), aunque es necesario comentar que en diferente medida todos los tipos de asociaciones ofrecen este tipo de formación, siendo las voluntaristas de base las que menos se implican en este tipo de cursos.

\section{Organización de sesiones de sensibilización externa}

Es una de las actividades que más organizan las asociaciones (la segunda en importancia: un 30,6\% de estas dice realizar acciones de este tipo). Las asociaciones que llevan más años creadas son las que más actividades de este tipo realizan (53,8\%). Por género se realizan en todos los tipos de asociaciones, pero el tipo de asociación que más las realiza es el de las asociaciones formadas principalmente por hombres $(35,4 \%)$. Por zonas de origen las tres tipologías de asociaciones tienen porcentajes muy similares 
en la organización de estas actividades. Así mismo, las asociaciones que hemos definido como profesionalizadas son las que organizan más actividades de sensibilización externa $(63,6 \%)$, junto con las voluntaristas reticulares $(55,8 \%)$.

\section{Actividades de acogida}

Si bien las actividades bajo el concepto de acogida no tienen por qué presentar unas particularidades educativas, las incluimos en este estudio debido a que en la Comunidad Valenciana algunas asociaciones de inmigrantes (un $11 \%$ de las asociaciones valencianas dicen llevar a cabo acciones de acogida) impulsan entre sus actividades "Escuelas de acogida", una actividad que responde al desarrollo del "Programa Voluntario de Comprensión de la Sociedad" que tiene como objetivo formar a las personas de origen inmigrante en la lengua castellana y valenciana; en legislación básica (Constitución española, Estatuto de autonomía...); Geografía e Historia de España y de la Comunidad Valenciana y también en temas de extranjería, ocupación, vivienda y sanidad. Este curso tiene una duración de cuarenta horas y superarlo supone puntos a favor para obtener la regulación por la vía de enraizamiento.

“- El tema del aula de acogida, me habían comentado que teníais. ¿Cómo funciona? ¿Viene gente de Senegal solamente o viene...?

- No, está abierta a todo el mundo, a todos los inmigrantes. La última clase terminó el sábado pasado. Había muchas nacionalidades también, no solamente de Senegal. Había marroquíes, argelinos, gente de Europa del Este y otras nacionalidades también. Y también hacemos clases de español, que hacemos aquí también.

- ¿Y cómo valoras el aula de acogida?

- Pues yo personalmente tengo dos lecturas. Hay una que es un poco política. Yo sé que hay un poco de política en esto, porque eso fue uno de los puntos del programa electoral de Mariano Rajoy, entonces, ellos lo han aplicado. Y otra cosa, está bien por los conocimientos, porque se les enseñan lo del cuaderno, un poco de los servicios de la ciudad, por ejemplo, los diferentes servicios, el idioma español, que es muy importante, un poco de valenciano, un poco de leyes. Eso es el lado positivo que veo yo, [...] pero por otro lado ya te digo que es un poco político" (ASEHA10).

\section{Clases de lengua de origen}

Una de las actividades más habituales. Cuando se trata de clases de lengua o cultura de origen, se esgrimen fines de carácter identitario (voluntad de mantener la "propia" lengua o cultura), aunque no exclusivamente, pues también pueden darse argumentos de carácter instrumental:

"Nuestro objetivo era para que los niños aprendan a hablar sus idiomas, porque cuando van a su país tienen problemas, no saben hablar con la familia, tienes que estar traduciendo" (AMAMT10I). 
Por los datos obtenidos es una actividad formativa que está consolidada en asociaciones que llevan años constituidas. Se imparten menos en las asociaciones subsaharianas $(5,3 \%)$ frente al $17,6 \%$ en las mixtas o el $12,9 \%$ en las norteafricanas. Por género, el tipo de asociación que más organiza estas clases es el de las asociaciones formadas solamente por mujeres. Esta formación es impartida por todas la tipologías de asociaciones, siendo las profesionalizadas $27,3 \%$ y las voluntaristas organizadas $(15,8 \%)$ las asociaciones que más las organizan.

Clases de cultura de origen

Si tenemos en cuenta los tipos de asociaciones según la composición de género, observamos que esta modalidad de clases no se organiza en las asociaciones que están formadas únicamente por mujeres, pero sí en las otras tipologías de asociaciones según el género. Si observamos las asociaciones en función de las zonas de origen, vemos que las clases de cultura de origen se imparten más desde las asociaciones norteafricanas $(21 \%)$ y mucho menos desde las subsaharianas (3,9\%). Las cuatro tipologías de asociación que hemos creado imparten estas clases. El 27,3\% de las asociaciones profesionalizadas las llevan a cabo y también el 19,3\% de las voluntaristas estructuradas. Estos porcentajes disminuyen de forma considerable en el caso de las asociaciones voluntaristas de base (el 6,3\%) y voluntarista reticular (el 4,7\%)

Conviene, sin embargo, introducir un matiz que vale tanto para este tipo de actividades formativas, como para los otros que hemos mencionado: esta relación hace referencia exclusivamente a los cursos o las clases que estas asociaciones organizan. No tiene en cuenta, sin embargo, que tales actividades educativas se pueden desarrollar a través de actividades de carácter más informal. Y si tenemos en cuenta estas actividades más informales, algunas de las correlaciones observadas se podrían llegar a cuestionar. Por ejemplo: en algunas asociaciones integradas exclusivamente o mayoritariamente por mujeres de origen subsahariano el repertorio de actividades de carácter formativo (y eminentemente dirigidas a niños) se amplía al relato de cuentos y a los juegos de origen africano:

"- ¿Y vosotras con los niños hacéis algo para transmitir vuestra cultura desde la Asociación?

- Sí, a partir de Junio, con cuentos. Ella que es una madre para nosotras, y a partir de aquí porque en África los cuentos están para enseñar los valores de la sociedad, para ser buenas personas; entonces hemos pensado hacer tardes de cuentos para nuestros niños" (ABFMG10).

Así pues, efectivamente, entre estas asociaciones no se organizan "clases" de cultura de origen, lo cual no quiere decir que entre estas asociaciones esta cuestión no sea atendida. Aparentemente, la preocupación por los niños, jóvenes y adolescentes se hace más aguda entre los actores que más protagonismo toman en relación a la repro- 
ducción cultural de sus comunidades (dentro de la familia, las mujeres; y de un modo muy distinto, los responsables religiosos). En torno a esa cuestión (la reproducción cultural, el mantenimiento de las lenguas y culturas de origen) encontramos abundantes referencias a los menores y a las iniciativas educativas de las asociaciones. De hecho, los menores (niños, adolescentes, jóvenes) son la población-diana de los discursos de reproducción y a la vez, en situación de inmigración, son la población más sensible a los procesos de ruptura con la tradición. Estas son algunas de las referencias extraídas de las entrevistas a los responsables de las entidades de carácter religioso:

"¿Cuál es el motivo principal cuando surge la Asociación?

Para la cultura, para guardar nuestra cultura para no perder porque tenemos los niños aquí, para darles cursos de árabe. Para que la gente no va a perder su cultura" (AMAHN10II).

"- Muy bien, ¿me podrías explicar los objetivos y la filosofía de vuestra asociación? ¿Cómo os definís? o ¿cuáles son los objetivos por los cuáles creasteis la asociación? 0 ¿cuáles son los valores de la asociación? ¿Qué queréis transmitir o qué queréis?

- Mmmmm..... Es la integración. Primeramente es la integración. Más que no perdamos las costumbres. Lo que pasa que hay mucha gente que ya vienen aquí y es otro mundo y se pierde. Pero no queremos perder nuestras costumbres y también la religión" (ASEHLL10).

Desde otro tipo de asociaciones se hace referencia a los jóvenes (por ejemplo en las entrevistas a las asociaciones de estudiantes y en las realizadas a los diferentes representantes de Ibn Batuta), mucho menos a los niños, pero es sobre todo en las entrevistas a las asociaciones de mujeres y en las de carácter religioso (islámico), en las que estas referencias aparecen estrechamente vinculadas a la preocupación por el mantenimiento y/o reproducción cultural.

\section{Formación externa en la que participan las asociaciones}

Más allá de los cursos, clases y sesiones que las asociaciones promueven y que ofrecen a sus asociados 0 a sectores de población más amplios, es habitual que las asociaciones participen en actividades de formación, educación y sensibilización organizadas por otros, ya sean escuelas, ayuntamientos, otras organizaciones no gubernamentales, fundaciones...

"Participamos, por ejemplo, con la CAM. La CAM organiza también encuentros interculturales, o charlas, debates en las aulas, y participamos siempre. De hecho, el año pasado hemos recibido un premio de la CAM como mejor asociación en Alicante. Y participamos también en actividades de otras asociaciones de inmigrantes" (ASEHA10). 
Algunas de las actividades realizadas por las asociaciones a propuesta de otras entidades 0 en el marco de iniciativas de otras organizaciones, tienen un carácter más marcadamente educativo. Lo tienen sobre todo cuando las actividades se organizan en o para las escuelas. En los centros escolares es habitual que se ofrezcan espacios de participación para las asociaciones de inmigrantes. Básicamente hemos detectado tres tipos de actividades para las que se requiere a las asociaciones (o a miembros destacados de las asociaciones):

1. La participación en encuentros interculturales (tomamos la denominación que habitualmente se utiliza).

2. La realización de charlas, conferencias y debates.

3. El apoyo a la escuela participando en dinámicas de mediación, refuerzo escolar, traducción, acompañamientos, entre otros.

Los dos primeros tipos de nuevo se sitúan en la frontera entre lo que consideramos formación y sensibilización. Sin embargo, en la medida en que tales actividades se desarrollan en contextos de educación formal, pensamos que debemos considerarlas actividades plenamente educativas y que debemos analizarlas desde ese punto de vista.

"Ahora con el tema del velo tenemos que intentar trabajar este tema en colegios, asistir a conferencias, dar charlas, cosas interculturales, meriendas..." (AMAMV10).

Debemos considerar que las actividades de estas asociaciones (sean presentadas como actividades formativas o de sensibilización) contribuyen a dar forma al imaginario intercultural: qué es un determinado colectivo, qué significan las distintas prácticas, cómo se deben abordar los problemas interculturales.

¿Cuál es el papel o el uso que se hace de las asociaciones en los centros escolares? Por una parte observamos como en algunos casos los centros escolares ofrecen espacios en los que se transmite el mensaje de carácter reivindicativo 0 social de algunas asociaciones, pero en otros casos los espacios que se crean son plataformas de multiculturalismo en las que se muestra la diversidad existente en nuestra sociedad (la versión multiculturalista de esa diversidad) y la participación en charlas y debates no se aleja mucho de la participación en los encuentros de carácter multicultural más ortodoxos (realización de talleres, participación en semanas interculturales, etc.). En estos casos, se observa una marcada tendencia al desarrollo de actividades de carácter folclórico y a sumarse a dinámicas propias del multiculturalismo más estereotipado:

"Y como comentaba, en interculturalidad no nos dedicamos solamente a la danza, a la percusión. Hacemos gastronomía africana, hacemos platos típicos de los países" (ASEHV10). 
Pensamos que es preciso valorar esta tendencia al multiculturalismo de carácter más folclorista. La crítica al multiculturalismo más estereotipado es ya un lugar común en muchos ámbitos y especialmente en el ámbito de la educación. De él se ha criticado su tendencia a la estereotipación, pero también su inocuidad frente a los discursos del nuevo racismo 0 incluso su capacidad de alimentarlos (Delgado 2003; Serra 2002a). Estos planteamientos, ya plenamente asumidos por la pedagogía, la sociología y la antropología de la educación, deben tenerse en cuenta en este nuevo contexto que estamos analizando. Aun tomando en consideración las prevenciones que más arriba expresábamos (de nuevo: no estamos analizando el ejercicio profesional de personas en instituciones con objetivos bien definidos, sino la práctica de asociaciones que en su mayoría son de carácter voluntario con motivaciones, objetivos e intereses muy diversos y no siempre explicitados), pensamos que a la hora de valorar la práctica de las asociaciones debemos reconocer sus consecuencias indeseadas. Nos atrevemos a citar tres: el empobrecimiento del discurso de las asociaciones, la estereotipación que conlleva y la neutralización de los discursos sociales más críticos o relevantes que podríamos esperar de este tipo de asociaciones:

"Y queríamos hacer también una manifestación grande, una fiesta de Senegal, para enseñar nuestra cultura en Gandía" (ADEMV10II).

"Cada uno sabe lo que le toca hacer culturalmente. Lo único que tenemos que presentar nosotros aquí es nuestra cultura y nuestra gastronomía, pero nada más. Por ejemplo, hay algunos que han sido profesores en Senegal, pero aquí no..." (ASEMT10).

La desactivación del discurso social que conlleva este discurso se hace más dramática si consideramos que la cita anterior está extraída de la entrevista al representante de una asociación de inmigrantes de origen subsahariano que se denomina asociación de inmigrantes "de lucha contra la pobreza".

Esta crítica es asumida por algunos de los representantes que hemos entrevistado, si bien han sido mayoría los entrevistados que no han mostrado dudas ante la deriva folclórica/multicultural de sus asociaciones (y que probablemente no se han reconocido en ella). El único discurso explícitamente crítico ante los tópicos del multiculturalismo lo hemos recogido de uno de los representantes de lbn Batuta, en Lleida:

\footnotetext{
¿QQué queremos exactamente? ¿Qué temas queremos tratar? ¿La asociación qué quiere dar a la inmigración? ¿Qué... qué discurso crítico tenemos sobre las asociaciones y nosotros no tenemos que caer en estos errores? O para superar los errores que nosotros vemos que las otras asociaciones no trabajan sobre temas que son, son muy interesantes, como la mujer, la juventud... Y también la manera de tratarte. Porque la mayoría de asociaciones ahora, hay asociaciones religiosas y hay otras que no, pero estas que no, no trabajan sobre temas que... en relación directa con la inmigración... Trabajan sobre el tema de la gastronomía, del vestido, del folclore. Y esto sobre... Yo, mi punto de vista, esto no sirve para nada para los inmigrantes" (AMAMLL10).
} 
Más allá de estas actividades, las asociaciones también participan en iniciativas de apoyo a las escuelas de carácter más instrumental. Algunas asociaciones son un punto de apoyo para algunas iniciativas escolares. $Y$ sin duda esto es positivo siempre y cuando el sumarse a dinámicas ajenas no impida que ellas mismas puedan desarrollar un discurso y actividades coherentes con sus objetivos (excepto en casos como el de Wafae, mencionado anteriormente, el apoyo a las iniciativas escolares no se encuentra entre los objetivos para los que se crearon de las asociaciones).

De manera absolutamente dominante, la dinámica de relación que se establece entre las asociaciones de personas inmigradas de origen africano y las escuelas es de cooperación. Un tipo especial de cooperación: las asociaciones dan apoyo a las escuelas en todo aquello que estas precisan y las asociaciones pueden facilitar. Y es que por lo general, el discurso lanzado desde las asociaciones sobre el ámbito educativo no es un discurso crítico. Con algunas excepciones que, aunque minoritarias, pensamos que merece la pena que reseñemos (y serán otros los que deban decidir si lo que en ellas se expone refleja situaciones minoritarias, o si lo minoritario es la voluntad, la resolución o la capacidad de exponer y denunciar tales situaciones). Por ejemplo, el relato de dos mujeres de origen subsahariano que justifican sus actividades de promoción de la cultura africana de sus respectivas culturas nacionales y de África en general como respuesta a la imagen que de ellas se da en las escuelas:

"Nuestros hijos, les queremos..., les queremos..., eh..., que conozcan nuestra cultura, entonces, nuestras actividades... Eh, hay muchas... Es cultura guineana, lo que damos. En Guinea Ecuatorial hay varias etnias, entonces, entre las actividades solemos hacer cursos sobre cultura guineana: viene un representante de cada etnia, te cuenta..., eh..., los problemas que tenemos... Nuestros hijos están acostumbrados a que en el colegio se ridiculice todo lo que sea negro, todo lo que sea africano, entonces los llevamos a esos sitios para que vean que no es tan malo" (AGUMV09).

- ¿YY vosotras con los niños hacéis algo para transmitir vuestra cultura desde la Asociación? - Sí, a partir de Junio, con cuentos. Hemos pensado hacer tardes de cuentos para nuestros niños.

- Claro, que bonito.

- Y otro tema es que están dando una idea de África en la escuela que no es real. Por ejemplo que dicen todo lo que es negativo de África y el niño lo aprende en la escuela y viene y te lo dice, y tú tienes que decirle "mira, no todo es negativo en África". No todos son pobres y no tienen para comer. Le dicen que no se lavan, que por eso son negros. Y eso hace daño. $O$ "tú no has comido".

- Vale, ¿que hay actitudes racistas con los niños?

- Racista es demasiado pero discriminatorias si" (ABFMG10).

En ambos ejemplos no se habla únicamente de reproducir (o recrear) las culturas de origen, sino de oponerse o de resistir a la visión que de ellas se tiene en los centros escolares. De un modo similar, en algunas asociaciones de mujeres se ha manifestado 
la necesidad de luchar contra los estereotipos que envuelven bien a las mujeres africanas, bien a las mujeres musulmanas:

"Se lucha muchísimo desde la asociación de mujeres para romper esos estereotipos cara a la sociedad, de demostrar que la mujer musulmana es un miembro activo, que tiene un nivel educativo bastante alto, que puede defenderse, que puede hablar por sí misma, que en realidad el velo nunca ha sido un acto de sumisión, sino es ella la que elige..." (AMAHN10).

\section{CONCLUSIONES}

Como hemos expuesto, la formación es un valor importante para los dirigentes de las asociaciones que hemos entrevistado, ya que en algunos casos los estudios forman parte de su proyecto migratorio. Por otro lado observamos que los niveles de formación que presentan los dirigentes es elevado, casi la mitad tiene estudios superiores y un porcentaje bastante alto tiene estudios de bachillerato o de formación profesional. Solo un bajo porcentaje de dirigentes no ha superado los estudios básicos.

También hemos puesto de relieve que la formación es una parte muy importante de las actividades que organizan las asociaciones y que las asociaciones no solamente las organizan, sino que con cierta frecuencia son llamadas por diferentes organizaciones o entidades para que participen en actividades de sensibilización y difusión de las lenguas y culturas de origen, entre otros aspectos. En este sentido hemos puesto de relieve como el paradigma multiculturalista domina buena parte de sus acciones formativas. Un paradigma que conlleva el empobrecimiento del discurso de algunas asociaciones, una tendencia a la estereotipación y la neutralización de los discursos sociales más críticos. Todo ello es importante si las asociaciones quieren trabajar en una perspectiva inclusiva e intercultural.

Si nos centramos en el papel que realizan las diferentes tipologías de asociaciones según su organización y estructura observamos que las cuatro tipologías se muestran muy activas organizando actividades formativas, aunque se ha puesto de relieve que las asociaciones profesionalizadas son las que generan mayor oferta formativa.

Para finalizar, una cuestión que será necesario valorar en próximos estudios es hasta qué punto la oferta formativa que realizan las asociaciones es debida, principalmente, a la necesidad de generar actividades para dar sentido a la asociación y servicio a los asociados y comunidad (y se hacen actividades formativas como podrían hacerse actividades de cualquier otro tipo), o si el desarrollo de estas actividades responde a la necesidad de formación de estos colectivos y a la necesidad de crear espacios de comunicación y de identificación cultural, frente a la potente máquina asimiladora que en general es la sociedad de recepción. Si fuera la segunda opción podríamos recorrer un largo camino en la mejora de la oferta formativa de estas asociaciones. 


\section{RefERENCIAS BiBLIOGRÁfICAS}

Abella, M. 2006. "Competencia global por trabajadores cualificados". Pp. 185-205 en Migraciones. Nuevas movilidades en un mundo en movimiento, editado por C. Blanco. Barcelona: Anthropos.

Aparicio, R. y R. Tornos. 2010. Las asociaciones de inmigrantes en España. Una visión de conjunto. Madrid: Ministerio de Trabajo e Inmigración.

Blanco, C. 2006. Migraciones. Nuevas movilidades en un mundo en movimiento. Barcelona: Anthropos.

Colectivo loé y F. Fernández. 2010. Encuesta nacional de inmigrantes 2007: el mercado de trabajo y las redes sociales de los inmigrantes. Madrid: Ministerio de Trabajo e Inmigración.

Crespo, R. 1997. "Associacionisme immigrant". Pp. 321-340 en II Informe immigració i treball social, VV.AA. Barcelona: Diputació de Barcelona.

Delgado, M. 2003. Inmigración y cultura (Ciudad e inmigración II). Barcelona: Centre de Cultura Contemporània de Barcelona.

Franzé, A. 2002. Lo que sabía no valía. Escuela, diversidad e inmigración. Madrid: Consejo Económico y Social de la Comunidad de Madrid.

Franzé, A. 2008a. "Discurso experto, educación intercultural y patrimonialización de la 'cultura de origen'”. Pp. 61-89 en ¿Es la escuela el problema? Perspectivas socio-antropológicas de etnografía y educación, editado por M. I. Jociles y A. Franzé. Madrid: Trotta.

Franzé, A. 2008b. "A la sombra del origen: lengua, cultura e identidad en los fundamentos de la ELCO". Pp. 260-274 en ¿Es la escuela el problema? Perspectivas socio-antropológicas de etnografía y educación, editado por M. I. Jociles y A. Franzé. Madrid: Trotta.

Freire, P. 1990. La Naturaleza política de la educación: cultura, poder y liberación. Barcelona: Paidós.

Garreta, J. 1998. "Minories ètniques, associacionisme i integració sociocultural". Revista Papers 56:197-230.

Garreta, J. y N. Llevot. 2013. "Las asociaciones de inmigrantes africanos. Organización, proyección y actuaciones". Revista Internacional de Sociología, Vol. 71, Extra 1: 15-38

Giró, J. 2011. "Educación y formación profesional entre los dirigentes de asociaciones africanas de inmigrantes". Presentado en XV Conferencia de Sociología de la Educación, Granada.

Giroux, H. A, 2001. Cultura, política y práctica educativa. Barcelona: Graó.

Instituto Nacional de Estadística. 2008. Ministerio de Economía y Competitividad. Secretaría de Estado de Economía y Apoyo a la Empresa. Documentos de trabajo, 2/08. Encuesta nacional de inmigrantes 2007. Madrid: INE.

Moncusí, A. y M. Albert. 2013. "El rol del asociacionismo de inmigrantes africanos en la construcción de cohesión social y la convivencia en Cataluña, Navarra y la Comunidad Valenciana. Miradas cruzadas". Revista Internacional de Sociología, Vol. 71, Extra 1: 39-65.

Moreras, J. 2009. Actors i representacions. L'associacionisme d'origen marroquí a Catalunya. BarceIona: Generalitat de Catalunya. 
Pazos, A. 1998. "La representación de la cultura. Museos etnográficos y antropología". Política y Sociedad 27:33-45.

San Román, T. 1996. Los muros de la separación. Ensayo sobre heterofobia y filantropía. Madrid: Tecnos.

Serra, C. 2002a. "El Racismo observado. Etnografía y análisis de las relaciones interétnicas en el ámbito de la educación". Cuadernos de pedagogía 315:77-82.

Serra, C. 2002b. Antropologia de l'educació. L'etnografia i l'estudi de les relacions interètniques en l'àmbit de l'educació. Girona: Publicacions de la Universitat de Girona.

Soriano, E. y A. J. González. 2010. "El poder educativo de las asociaciones de inmigrantes en las escuelas multiculturales". Relieve 16:1-20. Consultado 21 de octubre de 2011 (http://www.uv.es/ RELIEVE/v16n1/RELIEVEv17n1_3.htm).

Josep Miquel Palaudàrias Martí es doctor en pedagogía y profesor de la Universidad de Girona, sus trabajos se centran en el estudio de las migraciones internacionales y la educación. Entre sus publicaciones destaca como coeditor La migración extranjera en España: balance y perspectivas (CCG, 2007) y es coautor de Entre l'abandonament i la continuïtat. Els alumnes membres de famílies immigrades davant el final de l'educació secundària obligatòria (Mediterrània 2010). En la actualidad está investigando sobre las relaciones entre las familias inmigradas y la escuela, y sobre los procesos de participación y democracia en las escuelas.

Carles Serra Salamé es antropólogo especializado en el análisis de la presencia de la población inmigrante en el sistema educativo. Entre sus publicaciones destaca Antropologia de l'educació. L'etnografia i l'estudi de les relacions interètniques en l'àmbit de l'educació (Univ. de Girona, 2002), Diversitat, racisme i violència. Conflictes a l'educació secundària (Eumo, 2006) y junto, a Josep Miquel Palaudàrias, Entre l'abandonament i la continuïtat. Els alumnes membres de famílies immigrades davant el final de l'educació secundària obligatòria (Mediterrània 2010), así como artículos sobre sobre cuestiones educativas relacionadas con la inmigración.

\section{RECIBIDO: 28/09/2012}

ACEPTADO: 18/04/2013 